\title{
MODELLING THE EFFECT OF FIBRE LENGTH DISTRIBUTIONS IN SHORT FIBRE REINFORCED COMPOSITES
}

\author{
$\underline{\text { N. Mentges }}{ }^{1,2^{*}}$, S.M. Mirkhalaf ${ }^{3}$, and M. Fagerström ${ }^{2}$
}

\author{
${ }^{1}$ Institute for Plastics Processing, RWTH Aachen University, Aachen, Germany \\ ${ }^{2}$ Dept. Industrial and Materials Science, Chalmers University of Technology, Gothenburg, Sweden \\ ${ }^{3}$ Department of Physics, University of Gothenburg, Gothenburg, Sweden \\ *noah.mentges@rwth-aachen.de
}

The mechanical behaviour of Short Fibre Reinforced Composites (SFRCs) is dependent on several different micro-structural parameters. A major influence on the mechanical response of SFRCs is caused by the fibre orientation distribution (FOD) and the geometrical aspects of reinforcements, which are represented by the fibre diameter and fibre length distribution (FLD). Both the FLD and the FOD vary locally in an injection moulded part [1]. For a reliable prediction of the material behaviour, these parameters need to be represented adequately in a micro-mechanical model [2]. In this work, the two-step Orientation Averaging (OA) model for SFRCs, developed by Mirkhalaf et al. [3], is extended to consider also FLD for linear elastic and non-linear elasto-plastic behaviour of SFRCs.

In the original model, the first step is to obtain the homogenised mechanical response of a single fibre Unit Cell (UC) by performing Finite Element Analyses. In the linear elastic case, a homogenised stiffness tensor is obtained. For the elasto-plastic case, a transverse isotropic surrogate model is calibrated to the UC response, using Hill's yield criterion with isotropic hardening. Including the fibre length distribution into the OA process is realised by adding an averaging scheme over the range of fibre lengths. The distribution of fibre lengths is represented by a volume weighted probability density function. Using this method results in good predictions of the homogenised mechanical response. However, the demand for several numerical simulations is a great disadvantage of this method.

Using a single representative fibre length instead, is not a novel approach in modelling SFRCs. Conventional methods using the number or volume averaged fibre length [4], however, neglect the nonlinear relation between fibre length and the composite stiffness. Considering the fibre parallel UC-stiffness as a weighting factor for obtaining an average fibre length takes these effects into account. The novel approach is referred to as the stiffness averaged fibre length.

In this work both described methods, using an additional averaging scheme and deriving a single representative fibre length, are presented and predictions obtained with these models are compared to experimental results from the literature. Both models show very good prediction capabilities in the linear elastic and the elasto-plastic domain. The model predictions are very similar, with variations of less than $3 \%$. Hence, using single representative fibre lengths is found beneficial due to its much better computational performance. Within the representative fibre lengths, the stiffness average usually gives the closest prediction to the other method.

\section{References}

[1] S.M. Mirkhalaf, E. Eggels, A.T. Anantharanga et al. (2019) Short fiber composites: Computational homogenization vs orientation averaging. ICCM22, Melbourne, Australia.

[2] K. Breuer, M. Stommel (2020) RVE modelling of short fiber reinforced thermoplastics with discrete fiber orientation and fiber length distribution. SN applied Sciences, 2, 91.

[3] S.M. Mirkhalaf, E. Eggels, T.J.H. van Beurden, et al. (2020) A finite element based orientation averaging method for predicting elastic properties of short fiber reinforced composites. Composites Part B, 202, 108388.

[4] P.J. Hine, H.R. Lusti, A.A. Gusev (2002) Numerical simulation of the effects of volume fraction, aspect ratio and fibre length distribution on the elastic and thermoelastic properties of short fibre composites. Composites Science and Technology, 62, 1445-1453. 